\title{
Universiteit
}

Leiden

The Netherlands

\section{Phase detection at the quantum limit with multiphoton Mach-Zehnder Interferometry}

Pezze, L.; Smerzi, A.; Khoury, M.J.; Hodelin, J.F.; Bouwmeester, D.

\section{Citation}

Pezze, L., Smerzi, A., Khoury, M. J., Hodelin, J. F., \& Bouwmeester, D. (2007). Phase detection at the quantum limit with multiphoton Mach-Zehnder Interferometry. Physical Review Letters, 99(22), 223602. doi:10.1103/PhysRevLett.99.223602

Version: $\quad$ Not Applicable (or Unknown)

License: $\quad$ Leiden University Non-exclusive license

Downloaded from: https://hdl.handle.net/1887/65881

Note: To cite this publication please use the final published version (if applicable). 


\title{
Phase Detection at the Quantum Limit with Multiphoton Mach-Zehnder Interferometry
}

\author{
L. Pezzé and A. Smerzi \\ BEC-CNR-INFM and Dipartimento di Fisica, Università di Trento, I-38050 Povo, Italy
}

G. Khoury, J. F. Hodelin, and D. Bouwmeester*

Department of Physics, University of California, Santa Barbara, California 93106, USA

(Received 22 January 2007; revised manuscript received 3 July 2007; published 27 November 2007)

\begin{abstract}
We study a Mach-Zehnder interferometer fed by a coherent state in one input port and vacuum in the other. We explore a Bayesian phase estimation strategy to demonstrate that it is possible to achieve the standard quantum limit independently from the true value of the phase shift and specific assumptions on the noise of the interferometer. We have been able to implement the protocol by using parallel operation of two photon-number-resolving detectors and multiphoton coincidence logic electronics at the output ports of a weakly illuminated Mach-Zehnder interferometer. This protocol is unbiased, saturates the CramerRao phase uncertainty bound, and, therefore, is an optimal phase estimation strategy.
\end{abstract}

DOI: 10.1103/PhysRevLett.99.223602

PACS numbers: $42.50 . \mathrm{St}$

The Mach-Zehnder (MZ) interferometer [1,2] is a truly ubiquitous device that has been implemented using photons, electrons [3], and atoms [4,5]. Its applications range from micro- to macroscales, including models of aerodynamics structures, near-field scanning microscopy [6], and the measurement of gravity accelerations [7]. The central goal of interferometry is to estimate phases with the highest possible confidence [8-10] while taking into account sources of noise. Recent technological advances make it possible to reduce or compensate the classical noise to the level where a different and irreducible source of uncertainty becomes dominant: the quantum noise. Given a finite energy resource, quantum uncertainty principles and back reactions limit the ultimate precision of a phase measurement. In the standard configuration of the MZ interferometer, a coherent optical state with an average number of photons $\bar{n}=|\alpha|^{2}$ enters input port $a$, and the vacuum enters input port $b$, as illustrated in Fig. 1. The goal is to estimate the true value of the phase shift $\theta$ after measuring a certain number of photons $N_{c}$ and $N_{d}$ at output ports $c$ and $d$, which, in the experiment discussed in this Letter, is made possible by two number-resolving photodetectors.

In contrast to a number of recent experiments exploring the Heisenberg limit [11], here we discuss phase sensitivity at the standard quantum limit. However, our estimation protocol, as well as our experimental detection capability, can be applied to explore a subshot-noise phase resolution once the interferometer is fed by nonclassical states of light $[8,9,12,13]$.

A common phase inference protocol for the scheme of Fig. 1 estimates the phase shift $\theta$ as $[12,14,15]$ :

$$
\Theta_{\text {est }}=\arccos \left(\frac{M_{p}}{\bar{n}}\right),
$$

where $M_{p}=\sum_{k=1}^{p}\left(N_{c}^{(k)}-N_{d}^{(k)}\right) / p$ is the photon-number difference detected at the output ports, averaged over $p$ independent measurements. The uncertainty of estimator (1) is

$$
\Delta \Theta=\frac{1}{\sqrt{p \bar{n}} \sin \theta},
$$

which follows from a linear error propagation theory. Equation (2) predicts an optimal working point at $\theta=$ $\pi / 2$, where the average photon-number difference varies most quickly with phase. As $\theta$ approaches 0 or $\pi$, the confidence of the measurement becomes very low and eventually vanishes.

As a consequence, this interferometric protocol does not allow the measurement of arbitrary phase shifts. This can be a serious drawback for applications such as laser gyroscopes, the synchronization of clocks, or the alignment of reference frames. Furthermore, to estimate small phase shifts with the highest resolution, the interferometer has to be actively stabilized around $\pi / 2$. This generally requires the addition of a feedback loop, which can be quite costly in terms of time and energy resources.

It was first noticed by Yurke, McCall, and Klauder (YMK) in [16] that the estimator (1) does not take into (a)

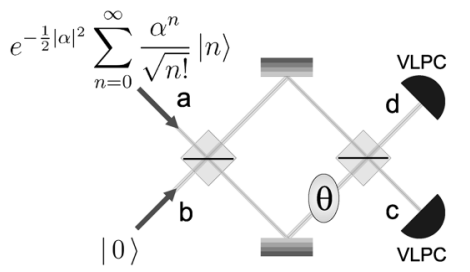

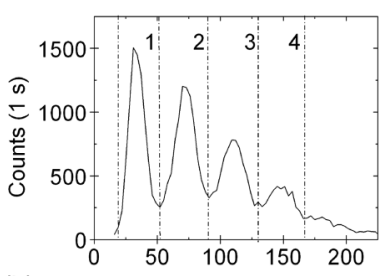

(b)

FIG. 1. (a) Schematic of a Mach-Zehnder interferometer. A phase sensitive measurement is provided by the detection of the number of particles $N_{c}$ and $N_{d}$ at the two output ports. (b) Pulse height distribution for a VLPC used in the experiment. The power incident on the detector is $144 \mathrm{fW}$ at a wavelength of $780 \mathrm{~nm}$. The vertical lines show the decision thresholds [23]. 
account all of the available information and, in particular, the fluctuations in the total number of photons at the output ports. The possibility to improve Eq. (2) is confirmed by the analysis of the Cramer-Rao lower bound (CRLB) [17], which provides, given an input state and choice of observables, the lowest uncertainty allowed by quantum mechanics. For an unbiased estimator, $\Delta \Theta_{\mathrm{CRLB}}=1 / \sqrt{p F(\theta)}$, where $F(\theta)$ is the Fisher information [17,18], which is additive and, in general, can depend on the true value of the phase shift $\theta$. An analytical calculation for the coherent $\otimes$ vacuum input state gives $F(\theta)=\bar{n}$ and

$$
\Delta \Theta_{\mathrm{CRLB}}=\frac{1}{\sqrt{p \bar{n}}},
$$

which, in contrast with the result of Eq. (2), is independent of the true value of the phase shift. The only assumption here is that the observable measured at the output ports is the number of particles. It is well known (see, for instance, [17]) that the maximum likelihood (ML) estimator, defined as the maximum $\Theta_{\mathrm{ML}}$ of the likelihood function $P\left(N_{c}, N_{d} \mid \phi\right)$ (see below), saturates the CRLB but only asymptotically in the number of measurements $p$. In the current literature, there have been alternative suggestions to obtain an unbiased estimator and a phase-independent sensitivity with a Mach-Zehnder interferometer [10,16,1921]. They will be specifically addressed at the end of this Letter.

Here we develop a protocol based on a Bayesian analysis of the measurement results $[8,9,17,19]$. The goal is to determine $P\left(\phi \mid N_{c}, N_{d}\right)$, the probability that the phase equals $\phi$ given the measured $N_{c}$ and $N_{d}$. The Bayes theorem provides this: $P\left(\phi \mid N_{c}, N_{d}\right)=P\left(N_{c}, N_{d} \mid \phi\right) P(\phi) /$ $P\left(N_{c}, N_{d}\right)$, where $P\left(N_{c}, N_{d} \mid \phi\right)$ is the probability to detect $N_{c}$ and $N_{d}$ when the phase is $\phi$ [22]. $P(\phi)$ quantifies our prior knowledge about the true value of the phase shift, and $P\left(N_{c}, N_{d}\right)$ is fixed by normalization. Assuming no prior knowledge of the phase shift in $[0, \pi], P(\phi)=1 / \pi$. In the ideal case, the Bayesian phase distribution can be calculated analytically for any value of $N_{c}$ and $N_{d}$ :

$$
P\left(\phi \mid N_{c}, N_{d}\right)=\mathcal{C}\left(\cos \frac{\phi}{2}\right)^{2 N_{c}}\left(\sin \frac{\phi}{2}\right)^{2 N_{d}},
$$

where $\mathcal{C}$ is a normalization constant. In practice, one must measure $P\left(N_{c}, N_{d} \mid \phi\right)$ and, from this, determine $P\left(\phi \mid N_{c}, N_{d}\right)$. This distribution provides both the phase estimate and the estimate uncertainty.

There are several advantages to using a Bayesian protocol. Notably, it can be applied to any number $p$ of independent measurements, it does not require statistical convergence or averaging, and it provides uncertainty estimates tailored to the specific measurement results. For instance, with a single measurement $p=1$, it predicts an uncertainty that scales as $\Delta \Theta \approx 1 / \sqrt{N_{c}+N_{d}}$. Since Eq. (4) does not depend on $\bar{n}$, the estimation is insensitive to fluctuations of the input laser intensity. Most impor- tantly, its uncertainty, in the limit $p \gg 1$, is $\Delta \Theta=$ $1 / \sqrt{p} \bar{n}$, which coincides with the CRLB. We note, however, that there is no proof that the Bayesian approach saturates the CRLB for all possible input states.

To implement the proposed protocol, we use an inherently stable, polarization Mach-Zehnder interferometer with photon-number-resolving coincidence detection. In a recent Letter, we reported on the analysis of a coherent state using a single photon-number-resolving detector [23]. We have extended this experimental capability to two simultaneously operating visible light photon counters (VLPCs) [24], cryogenic photodetectors that provide a current pulse of approximately 40000 electrons per detected photon. The VLPCs were maintained at $8 \mathrm{~K}$ in a helium flow cryostat, and their photocurrent was amplified by low-noise, room temperature amplifiers. We measured a detection efficiency of $40 \%$ and a dark count rate of $2 \times$ $10^{5}$ for each detector under our operating conditions. Custom electronics processed the amplified VLPC current pulses to perform gated, fast coincidence detection. We were thus able to determine, for each pulse, how many photons were detected at both ports $c$ and $d$. A Ti:sapphire pulsed laser, attenuated such that $\bar{n}=1.00$ (3) photons, provided the input state. Since a coherent state maintains its form under linear loss [14], the presence of loss after the interferometer is completely equivalent to a lossless interferometer fed by a weaker input state. We use $\bar{n}$ to signify the average number of photons in the detected state per pulse, after all losses. We were limited by the amplifiers to measuring up to four photons per pulse [Fig. 1(b)], but at $\bar{n} \approx 1$ the probability of detecting five or more photons is negligible. The phase shift $\theta$ was changed by tilting a birefringent crystal inside the MZ.

The first part of the experiment consists of the calibration of the interferometer. At 40 different values of the phase shift, we measured $N_{c}$ and $N_{d}$ for each of 200000 laser pulses. This procedure allows us to determine experimentally both $P_{\exp }\left(N_{c}, N_{d} \mid \phi\right)$ and $P_{\exp }\left(\phi \mid N_{c}, N_{d}\right)$. Note that these functions are defined only for the phases used during the calibration. In Fig. 2, we compare the ideal (solid line) and the experimental (circles) phase distributions. The discrepancies between ideal and experimental distributions can be attributed to imperfect photon-number discrimination. An advantage of the Bayesian protocol consists in the possibility to straightforwardly include in the analysis all of the detection noise.

After the calibration, we can proceed with the Bayesian phase estimation experiment. For a certain value of the phase shift $\theta$, we input one laser pulse and detect the number of photons $N_{c}$ and $N_{d}$. We repeat this procedure $p$ times, obtaining a sequence of independent results $\left\{N_{c}^{(i)}, N_{d}^{(i)}\right\}_{i=1 \ldots p}$. The $p$ photon-number measurements comprise a single phase estimation. The overall phase probability is given by the product of the distributions associated with each experimental result: 

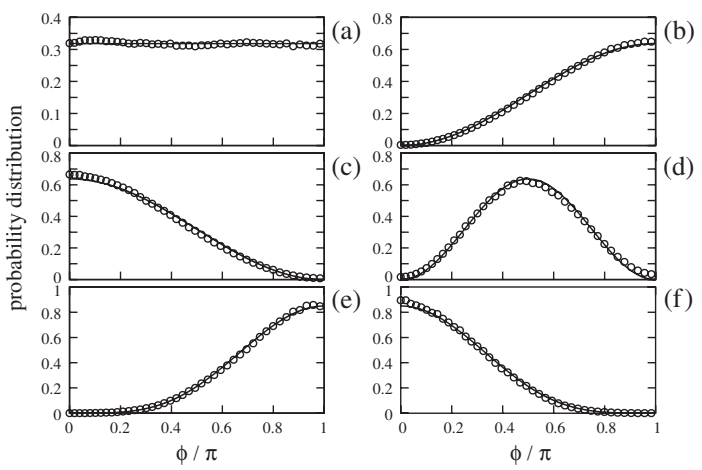

FIG. 2. Phase distribution $P\left(\phi \mid N_{c}, N_{d}\right)$, for: (a) $N_{c}=0, N_{d}=$ 0; (b) $N_{c}=0, N_{d}=1$; (c) $N_{c}=1, N_{d}=0$; (d) $N_{c}=1, N_{d}=$ 1 ; (e) $N_{c}=0, N_{d}=2$; (f) $N_{c}=2, N_{d}=0$. The circles are the experimental data collected in the calibration part of the experiment, and the solid line is the ideal distribution equation (4).

$$
P_{\exp }\left(\phi \mid\left\{N_{c}^{(i)}, N_{d}^{(i)}\right\}_{i=1 \ldots p}\right) \propto \prod_{i=1}^{p} P_{\exp }\left(\phi \mid N_{c}^{(i)}, N_{d}^{(i)}\right) .
$$

The phase estimator $\Theta_{\text {est }}$ is given by the mean value of the distribution $\Theta_{\mathrm{est}}=\int_{0}^{\pi} d \phi \phi P_{\mathrm{fit}}\left(\phi \mid\left\{N_{c}^{(i)}, N_{d}^{(i)}\right\}_{i=1 \ldots p}\right)$, where $P_{\text {fit }}\left(\phi \mid\left\{N_{c}^{(i)}, N_{d}^{(i)}\right\}_{i=1 \ldots p}\right)$ is a fit of the experimental distribution equation (5), and the phase uncertainty $\Delta \Theta$ is the $68.27 \%$ confidence interval around $\Theta_{\text {est }}$. Examples of $P_{\text {fit }}\left(\phi \mid\left\{N_{c}^{(i)}, N_{d}^{(i)}\right\}_{i=1 \ldots p}\right)$ are given in Figs. 3(a)-3(c) for $\theta / \pi=0.25$ and for different values of $p$. Since the average number of photons of the coherent input state is small, for $p \sim 1$ the phase uncertainty is of the order of the prior knowledge $\Delta \Theta \simeq \pi$. As $p$ increases, the distributions become nearly Gaussian, and the sensitivity scales as $\Delta \Theta \propto$ $1 / \sqrt{p}$ [see Fig. 3(d)], in agreement with the central limit theorem.
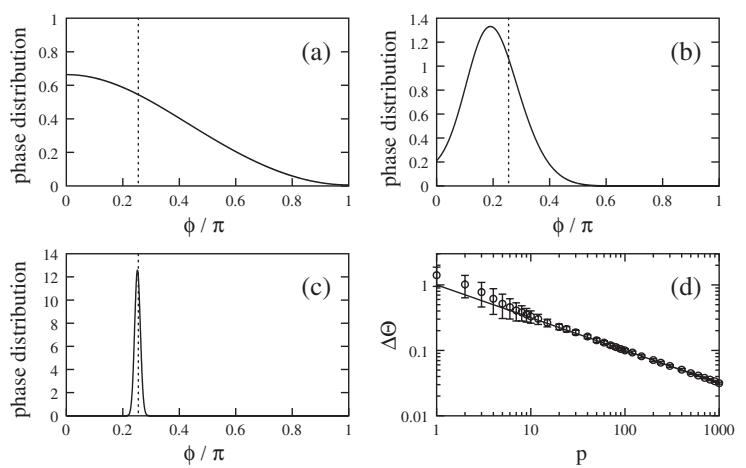

FIG. 3. Phase distribution $P_{\text {fit }}\left(\phi \mid\left\{N_{c}^{(i)}, N_{d}^{(i)}\right\}_{i=1 \ldots p}\right)$ obtained after $p$ independent experimental measurements: (a) $p=1$; (b) $p=10$; (c) $p=1000$. The true value of the phase shift is $\theta / \pi=0.25$, shown by the vertical dashed line. In (d), we plot $\Delta \Theta$ as a function of the number of measurements $p$. Circles are experimental results (averaged over 20 replicas of $p$ measurements, where error bars are mean square fluctuations), and the solid line is $\Delta \theta=1 / \sqrt{p}$, Eq. (3). The preasymptotic behavior $(p \lesssim 20)$ is due to a biased estimation.
The main result of this Letter is presented in Fig. 4. We show the phase sensitivity for different values of the phase shift $\theta$, calculated after $p=10000$ photon-number measurements. The circles are the mean value of $\Delta \Theta$, and the bars give the corresponding mean square fluctuation, obtained from 20 independent phase estimations. The dashed blue line is the CRLB calculated with the experimental probability distributions $\Delta \Theta_{\mathrm{CRLB}}^{\exp } \equiv 1 / \sqrt{p F_{\exp }(\theta)}$, where $\quad F_{\exp }(\theta)=\sum_{N_{c}, N_{d}}\left[1 / P_{\exp }\left(N_{c}, N_{d} \mid \theta\right)\right] \times$ $\left[\partial P_{\exp }\left(N_{c}, N_{d} \mid \theta\right) / \partial \theta\right]^{2}$. For $0.1 \lesssim \theta / \pi \lesssim 0.9$, it follows the ideal prediction (solid black line), Eq. (3). Around $\theta=$ $0, \pi$, where the photons have a higher probability to exit through the same output port, $\Delta \Theta_{\mathrm{CRLB}}^{\mathrm{exp}}$ increases as a consequence of the decreased ability of our detectors to distinguish higher photon-number states, but it does not diverge. The experimental results (circles) saturate $\Delta \Theta_{\mathrm{CRLB}}^{\mathrm{exp}}$. Squares show the phase uncertainty obtained with Eq. (1) but taking into account the experimental noise, which is obtained by inverting the equation $M_{p}=$ $a \cos (b+\theta)+c$ [25]. This strategy provides an unbiased estimation with a sensitivity close to the one predicted by Eq. (2) (dotted red line). The superior performance of the Bayesian protocol can be understood by noticing that, in Eq. (2), the phase estimate is retrieved from the measurement of the photon-number difference, which simply does not exploit all of the available information. In fact, by using the ideal probabilities to measure only the relative number

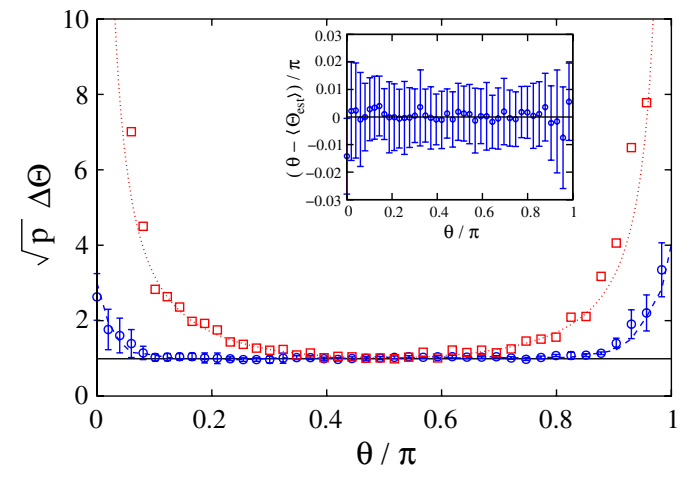

FIG. 4 (color online). Phase sensitivity as a function of the true value of the phase shift. Circles are obtained from the Bayesian distributions equation (5), with $p=10000$. The error bars give the fluctuations of $\sqrt{p} \Delta \Theta$ obtained with 20 independent replicas of the experiment. The solid black line is the theoretical prediction equation (3). The dashed blue line is the CRLB calculated with the experimental distributions. Squares are the uncertainty obtained with a generalization of the estimator equation (1) taking into account the experimental imperfections, while the dotted red line is the phase sensitivity predicted by Eq. (2). Inset: Difference between the mean value of the phase estimator $\left\langle\Theta_{\text {est }}\right\rangle$ obtained after 20 replicas of $p=10000$ independent measurements and the true value of the phase shift $\theta$. The vertical bars are the mean square fluctuations $\sigma_{\text {est }}^{2}=$ $\left\langle\left(\Theta_{\text {est }}-\left\langle\Theta_{\text {est }}\right\rangle\right)^{2}\right\rangle$. The $\sigma_{\text {est }} \gg\left(\theta-\left\langle\Theta_{\text {est }}\right\rangle\right)$ provides the evidence that our result is unbiased. 
of particles and calculating the corresponding CRLB [26], we recover exactly Eq. (2). In the inset in Fig. 4, we show the difference between the mean value of the phase estimator $\left\langle\Theta_{\text {est }}\right\rangle$ and the true value of the phase shift. The important result is that our protocol provides an experimentally unbiased estimation over the entire phase interval.

In Ref. [16], YMK first proposed a generalization of the estimator Eq. (1) to take into account the whole information in the output measurements. Their estimator $\Theta_{\mathrm{YMK}}=$ $\arccos \left[\left(N_{c}-N_{d}\right) /\left(N_{c}+N_{d}\right)\right]$ gives a phase-independent sensitivity $\Delta \Theta=1 / \sqrt{N_{c}+N_{d}}$ and saturates the CRLB. Notice that $\Theta_{\text {YMK }}$ coincides with the maximum likelihood estimator $\Theta_{\mathrm{ML}}$ in the ideal, noiseless, MZ interferometer. However, it is not obvious how to generalize $\Theta_{\text {YMK }}$ for real interferometry, where classical noise is present and $\Theta_{\mathrm{YMK}} \neq \Theta_{\mathrm{ML}}$. In general, because of correlations between $N_{c}-N_{d}$ and $N_{c}+N_{d}$, this estimator can become strongly biased in the presence of noise as we have verified using our experimental data. Moreover, the YMK estimator cannot be extended when both input ports of the interferometer are illuminated. Conversely, the Bayesian analysis holds for general inputs, and, in particular, it predicts a phaseindependent sensitivity also when squeezed vacuum is injected in the unused port of the MZ, which reaches a phase sensitivity at the Heisenberg limit [13].

In Ref. [19], Hradil et al. used a Bayesian approach for a Michelson-Morley neutron interferometer (single output detection) and discussed theoretically the MZ. Their analysis was based on specific assumptions about the interferometric classical noise which are not satisfied in the case discussed in this Letter. Different approaches with adaptive measurements [20] and positive operator value measurements [10] have also been suggested. While these strategies might be important for interferometry at the Heisenberg limit, they are not necessary in our case.

In conclusion, we have presented a Bayesian phase estimation protocol for a MZ interferometer fed by a single coherent state. The protocol is unbiased and provides a phase sensitivity that saturates the ultimate Cramer-Rao uncertainty bound imposed by quantum fluctuations. We have been able to implement the protocol with two photonnumber-resolving detectors at the output ports of a weakly illuminated interferometer. Yet the method can be generalized to the case of high intensity laser interferometry and photodiode detectors. In this case, the limit equation (3) becomes harder to achieve because of larger electronic noise and lower photon-number resolution; however, it should still be possible to demonstrate a phase-independent sensitivity. Our results are of importance to quantum inference theory and show that the MZ does not require phase-locking in order to reach an optimal sensitivity.

This work has been partially supported by the U.S. DOE and NSF Grant No. 0304678.
*Also at Huygens Laboratory, Leiden University, 2300 RA Leiden, The Netherlands.

[1] L. Mach, Z. Instrum.kd. 12, 89 (1892).

[2] L. Zehnder, Z. Instrum.kd. 11, 275 (1891).

[3] Y. Ji et al., Nature (London) 422, 415 (2003).

[4] Atom Interferometry, edited by P. R. Berman (Academic, New York, 1997).

[5] Y. Wang et al., Phys. Rev. Lett. 94, 090405 (2005); Y. Torii et al., Phys. Rev. A 61, 041602(R) (2000).

[6] F. Zenhausern et al., Science 269, 1083 (1995).

[7] A. Peters, K. Y. Chung, and S. Chu, Nature (London) 400, 849 (1999).

[8] M. J. Holland and K. Burnett, Phys. Rev. Lett. 71, 1355 (1993).

[9] L. Pezzé and A. Smerzi, Phys. Rev. A 73, 011801(R) (2006).

[10] B. C. Sanders and G. J. Milburn, Phys. Rev. Lett. 75, 2944 (1995).

[11] M. W. Mitchell, J.S. Lundeen, and A. M. Steinberg, Nature (London) 429, 161 (2004); P. Walther et al., Nature (London) 429, 158 (2004); T. Nagata et al., Science 316, 726 (2007).

[12] C. M. Caves, Phys. Rev. D 23, 1693 (1981).

[13] L. Pezzé and A. Smerzi, arXiv:0705.4631.

[14] M.O. Scully and M.S. Zubairy, Quantum Optics (Cambridge University Press, Cambridge, England, 1997).

[15] J. P. Dowling, Phys. Rev. A 57, 4736 (1998).

[16] B. Yurke et al., Phys. Rev. A 33, 4033 (1986).

[17] C. W. Helstrom, Quantum Detection and Estimation Theory (Academic, New York, 1976).

[18] The Fisher information is defined as $F(\theta)=$ $\sum_{N_{c}, N_{d}}\left[1 / P\left(N_{c}, N_{d} \mid \theta\right)\right]\left[\partial P\left(N_{c}, N_{d} \mid \theta\right) / \partial \theta\right]^{2}$.

[19] Z. Hradil et al., Phys. Rev. Lett. 76, 4295 (1996).

[20] D. W. Berry and H. M. Wiseman, Phys. Rev. Lett. 85, 5098 (2000).

[21] J. W. Noh, A. Fougeres, and L. Mandel, Phys. Rev. Lett. 67, 1426 (1991); J. Rehacek et al., Phys. Rev. A 60, 473 (1999).

[22] The quantum mechanical probability to detect $N_{c}$ and $N_{d}$ particles at the output port $c$ and $d$, respectively, is given by $P\left(N_{c}, N_{d} \mid \phi\right)=\mid\left(\left.\left\langle N_{c}\left|\left\langle N_{d}\right|\right) e^{-i \phi \hat{J}_{y}} \mid \psi_{\text {inp }}\right\rangle\right|^{2}\right.$, where $\hat{J}_{y}=$ $\left(\hat{a}^{\dagger} \hat{b}-\hat{b}^{\dagger} \hat{a}\right) / 2 i$, with $\hat{a}, \hat{b}$ bosonic annihilation operators. For the configuration studied in this Letter, $P\left(N_{c}, N_{d} \mid \phi\right)=$ $\left[|\alpha|^{2\left(N_{c}+N_{d}\right)} e^{-|\alpha|^{2}} /\left(N_{c}\right) !\left(N_{d}\right) !\right]\left(\sin \frac{\phi}{2}\right)^{2 N_{c}}\left(\cos \frac{\phi}{2}\right)^{2 N_{d}}$.

[23] G. Khoury et al., Phys. Rev. Lett. 96, 203601 (2006).

[24] G. B. Turner et al., in Proceedings of the Workshop on Scintillating Fiber Detectors, Notre Dame University, edited by R. Ruchti (World Scientific, Singapore, 1994).

[25] A fit of the calibration data gives $\left\langle N_{c}-N_{d}\right\rangle=a \cos (\theta+$ $b)+c$, with $a=0.98, b=0.007$, and $c=0.01$.

[26] The probability to detect a relative number of particles $N_{r}=N_{c}-N_{d}$ is $P\left(N_{r} \mid \theta\right)=\sum_{N_{c}, N_{d}} P\left(N_{c}, N_{d} \mid \theta\right) \delta_{N_{c}-N_{d}, N_{r}}$, and the corresponding Fisher information is $F_{r}(\theta)=$ $\sum_{N_{r}=-\infty}^{+\infty}\left[1 / P\left(N_{r} \mid \theta\right)\right]\left[\partial P\left(N_{r} \mid \theta\right) / \partial \theta\right]^{2}=\bar{n} \sin ^{2} \theta$. 